\title{
Mechanics and simulations of the groundsill damage due to the impacts from pebbles/cobbles
}

\author{
Y.-W. Pan, K.-P. Leong \& J.-J. Liao \\ Department of Civil Engineering, National Chiao Tung University, Hsinchu, Taiwan \\ C. S. Ku \\ Department of Civil and Ecological Engineering, I-SHOU University, Kaohsiung, Taiwan
}

\begin{abstract}
A pebble/cobble passing a head drop tends to carry a large impact momentum when it hits a groundsill; the heavy impact may result in the breakage of concrete. This study explores the mechanics and the principles of this issue by computational fluid dynamics with particle tracking. The results reveal that the kinematics of a single particle passing a head drop is primarily affected by the size of particles. A small particle tends to travel along the flow lines and brings relatively low impact energy. A large particle is more affected by gravity; saltation is more likely and will carry relatively high impact energy. The major role of the discharge magnitude is just to enable the transportation of larger particles to the downstream. With the simulation, it is possible to evaluate the impact position, load and kinetic energy. The locations prone to damage and failure potential can then be identified.
\end{abstract}

\section{GENERAL INSTRUCTIONS}

The outcrop in the west foothills of Taiwan is majorly composed of soft rock. Intense rock bed incision sometimes becomes a serious threat for the stability of a river channel and its nearby structures (Huang et al. 2012, 2013, 2014). The rivers in Taiwan are, in general, relatively short and steep. During very strong flows in a flood event, bedload with particle sizes varying, typically from gravel to cobbles (to be noted as "gravel" hereafter) can be transported to the midstream/ downstream of a river. Once the armour layer on the riverbed is eroded away, severe bed incision may be triggered so that it disturbs the stability of the river channel and endangers adjacent infrastructures. When necessary, groundsill may be installed to protect the riverbed from uncontrollable abrasion and impacts caused by the saltation of particles. The function of a groundsill is to protect the underlying riverbed from erosion, especially adjacent to a bridge or weir structure.

Compared to the bed stress induced by water flow, the collision with bedload, especially large gravel, is more likely to damage the revetment. The hardness of gravel is usually significantly higher than the revetment material which is usually made of concrete with low to medium strength. As a result, when big gravel collides the revetment, the concrete may be damaged because of excess impact stress from a concentrated dynamic load, and may eventually cause the failure of the revetment. The groundsill then may lose its function.

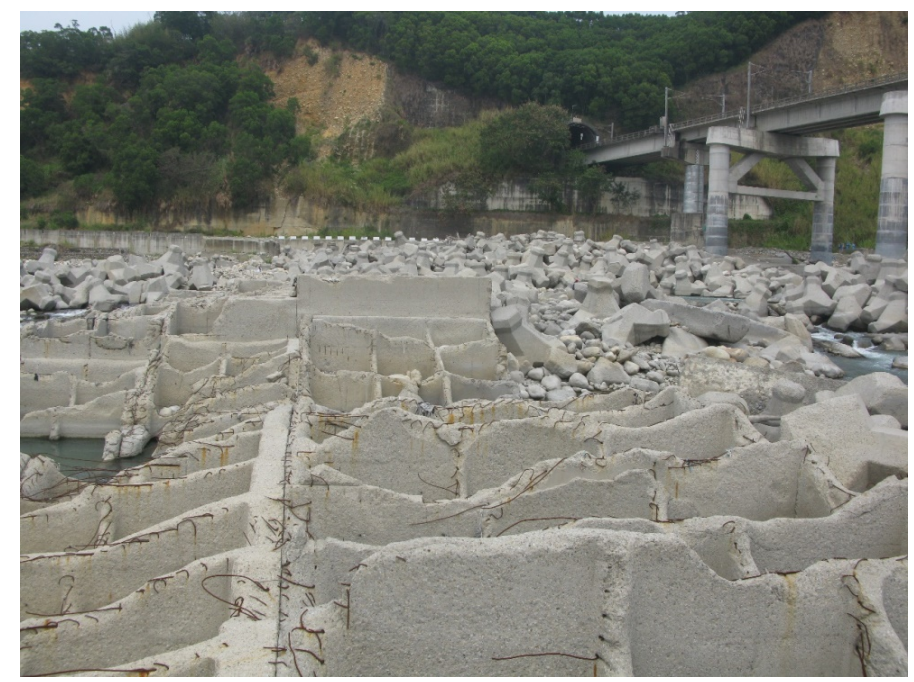

Figure 1. An example of groundsill damage due to impacts from particles.

Groundsills are often installed at downstream of a weir where the water level is elevated. In some cases, a stepped groundsill may be built to evenly house a head drop. During a strong discharge, large particles are able to be transported with the flow. When passing a head drop, a particle acquires its extra kinetic energy as a result of losing its potential energy. Once the attacking particle hit the downstream revetment or the river-bed armor, an instantaneous impact force will be generated at the contact point. The destructive behavior from the attacking particle may comprise of erosion peel, solid particle prising and abrasive wear (Plum \& Fang, 1996). 
When the dynamic contact stress exceeds the strength of concrete or surface rock, brittle failure in the concrete or the rock riverbed adjacent to the contact point can take place. The attacked material may also lead to failure because of accumulative fatigue as a result of the gradual increase of fissure network induced by sequential collisions (IKleis \& Kulu, 2008). The impact force and the contact stress may be assessed on the basis of the instantaneous particle velocity at the moment of impact using the classical Hertz theory of contact mechanics (Hertz, 1882). On the basis of the Hertz contact theory for the special condition of the contact between a sphere and a halfspace, the instantaneous contact force $F$ is proportional to $v_{\mathrm{o}}{ }^{1.2} R^{2}$, where $v_{\mathrm{o}}$ is the incipient particle velocity and $R$ is the radius of the attacking particle; the corresponding maximum pressure is $2 \pi a^{2}$, where $a$ is the dimension of contact. The terms $F$ and $a$ can be evaluated by analytical formula as long as $v_{\mathrm{o}}$ is known. If the maximum pressure exceeds the strength of concrete, the breakage of concrete will occur and lead to the failure of the revetment.

Large gravel particles can only travel downstream along with strong water current during a large hydrological event. To assess the potential for the damage of groundsill damaged by large particles, it is necessary to estimate the trajectory and the velocity of the moving particles. The present work made use of computational fluid dynamics (CFD) to numerically simulate the transportation of gravel particles. The purpose of the simulation is to track the particle path and to obtain the velocity of particles with various sizes.

The simulation records the impact variables of each gravel particle when the particle hits the river bed or revetment. The recorded impact variables include the particle coordinate, velocity and moving direction at the time of collision. With the calculated data, it is then possible to calculate the force induced by the particle collision using the concept of Hertz (1882), then to identify the damage caused by gravel particle with different sizes. The likelihood of concrete damage is evaluated by examining the normal force brought by the gravel particle collision.

\section{MODELLING APPROACH}

This work made use of the finite-element method (FEM) package "COMSOL Multiphysics" to conduct the simulation of particle movement along with turbulent flow in a flow channel. The software package COMSOL is capable of modelling coupled multi-physics problems by the finite-element method. The processes of numerical simulation in this work include two parts: (1) calculation of turbulent flow by CFD and (2) particle tracking

\subsection{Turbulent flow by CFD with moving grid}

For a high discharge capable of carrying large gravel particles, the flow condition in a river channel is often in a condition of turbulent flow. The CFD module of COMSOL is used to carry out the calculation of turbulent flow. Turbulent flow in an open channel is analyzed considering the governing equations including the mass conservation and the momentum conservation with the two-parameter $\mathrm{k}-\varepsilon$ turbulent model (COMSOL, 2013). The two equations for the mass conservation and the momentum conservation, respectively, are as follows.

$$
\begin{aligned}
& \rho \nabla \cdot u=0 \\
& \rho \frac{\partial u}{\partial t}+\rho(u \cdot \nabla) u=\nabla\left[-p \mathrm{I}+\mu\left(\nabla u+(\nabla u)^{T}\right)\right]+F
\end{aligned}
$$

For the $k-\varepsilon$ model, the variables $k$ and $\varepsilon$ stand for the turbulent kinetic energy and turbulent dissipation rate, respectively. There are two extra energy conservation equations: one for $k$ and the other for $\varepsilon$.

$$
\begin{aligned}
& \rho \frac{\partial k}{\partial t}+\rho u \cdot \nabla k=\nabla \cdot\left[\left(\mu+\frac{\mu_{T}}{\sigma_{k}}\right) \nabla k\right]+p_{k}-\rho \varepsilon \\
& \rho \frac{\partial \varepsilon}{\partial t}+\rho u \cdot \nabla \varepsilon=\nabla \cdot\left[\left(\mu+\frac{\mu_{T}}{\sigma_{\varepsilon}}\right) \nabla \varepsilon\right]-C_{\varepsilon 2} \rho \frac{\varepsilon^{2}}{k}+C_{\varepsilon 1} \frac{\varepsilon}{k} P_{k}
\end{aligned}
$$

In the above equations: $\rho=$ fluid density; $u=$ flow velocity; $p=$ fluid pressure; $F=$ volume force; $\mu=$ fluid viscosity; $P_{k}=$ production term in k-equation; $\sigma_{k}$ and $\sigma_{\varepsilon}=$ the turbulent numbers in the k-equation $\&$ in the $\varepsilon$-equation, respectively; $\mu_{T}=$ eddy viscosity; $C_{\varepsilon 1}$ and $C_{\varepsilon 2}=$ constants.

The water surface in an open channel is not known in advance before an analysis. To model the free water surface in an open channel problem, we used the Arbitrary Lagrangian-Eulerian Method (ALE) (Donea, et al. 1982) supported in COMSOL to model a moving grid for solving the free water surface. The condition on the free water surface has to satisfy the condition that the velocity component in the normal direction $n$ is zero. The moving grid is iteratively adjusted to meet the condition.

$$
u \cdot n=0
$$

\subsection{Particle Tracking}

The particle tracking module in the software package COMSOL enables the tracking of particle movement in a flow field. The forces acting on a particle considered in this work include the drag force, $F_{D}$ in water flow and the gravitational force, $F_{g}$; they can be expressed as follows 
$F_{D}=\frac{m_{p}}{\tau_{p}}\left(u-v_{p}\right)$

$F_{g}=m_{p} g \frac{\left(\rho_{p}-\rho_{w}\right)}{\rho_{p}}$

where the terms in the above equations are: $g=$ gravity acceleration; $\rho_{w}=$ water density; $v_{p}=$ particle velocity; $\tau_{p}=$ particle velocity response time. In a turbulent flow, $\tau_{p}$ can be evaluated by $\tau_{p}=\frac{4 \rho_{p} D_{p}{ }^{2}}{3 \mu C_{D} \operatorname{Re}_{r}}$, where $C_{D}=\frac{24}{\operatorname{Re}_{r}}\left(1+0.15 \operatorname{Re}_{r}{ }^{0.637}\right)$; the Reynolds number $\operatorname{Re}_{r}=\frac{\rho_{p}\left\|u-v_{p}\right\| D_{p}}{\mu}$.

The movement of a particle has to satisfy Newton's second law as follows.

$m_{p} \frac{d v_{p}}{d t}=F=F_{g}+F_{D}$

The rebound response of a particle hitting an obstacle wall has to follows Equation (9)

$v_{p 2}=v_{p 1}-2\left(n \cdot v_{p 1}\right) n$

in which $v_{p 1}$ and $v_{p 2}$ are the particle velocities before and after particle impact on the boundary wall, respectively. The normal and the tangential components of $v_{p 2}$ can be expressed by the following equations.

$$
\begin{aligned}
& v_{p 2_{n}}=\operatorname{COR}_{n}\left|v_{p 1}\right| \sin \alpha \\
& v_{p 2_{t}}=\operatorname{COR}_{t}\left|v_{p 1}\right| \cos \alpha
\end{aligned}
$$

where $C O R_{n}$ and $C O R_{t}$ are the normal and the tangential coefficients of restitution, respectively; $\alpha$ is the impact angle.

\subsection{Simulation Process}

A complete simulation starts with a two-dimensional turbulent flow analysis for an open channel representing a river section using the CFD module. In the CFD analysis for an open channel, we assign a prescribed discharge on the inlet. After the convergence of solution, the balanced flow field can be obtained. Subsequently, by adopting the resulting flow field from the CFD analysis, a particle tracking simulation can proceed by arbitrarily releasing particles from the flow inlet. The particle tracking module records the trajectory and the traveling velocity of each particle; it also records each gravel particle's variables when the particle hits the river bed or revetment. The recorded particle variables include the particle coordinate, the velocity and the direction of movement at the instance of collision. Once the variables of the particles' movement can be determined, further analysis of the potential of groundsill damage due to gravel strikes can be assessed.

\section{RESULT DEMONSTRATION - PARTICLES PASSING A HYPOTHETICAL WEIR}

A hypothetical case is used to demonstrate the approach of particle-movement simulation as described in the previous section. The hypothetical case is a flow channel containing a knickpoint with a head drop of $1.5 \mathrm{~m}$. Two conditions of unit discharge $q$, are modelled, one with $q=50 \mathrm{cms} / \mathrm{m}(1 \mathrm{cms}=1$ $\mathrm{m}^{3} / \mathrm{s}$ ), the other with $q=10 \mathrm{cms} / \mathrm{m}$; both cases are capable of carrying large gravel particles up to 400 $\mathrm{mm}$. Three different sizes of particle, $D$, are modeled: $D=400 \mathrm{~mm}, D=50 \mathrm{~mm}$ and $D=10 \mathrm{~mm}$. Figure 2 shows the flow lines and the velocity distribution obtained from the CFD computation. The maximum velocity can reach $12 \mathrm{~m} / \mathrm{s}$, approximately.

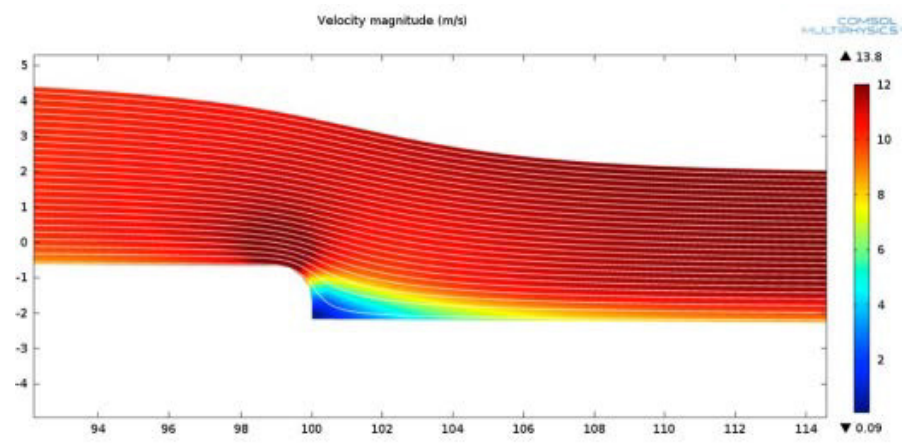

Figure 2. Flow lines and velocity distribution for $\mathrm{q}=50 \mathrm{cms} / \mathrm{m}$.

For the particle tracking simulation, the normal coefficient of restitution is assumed to be 0.7 . Figure 3 presents the results of particle tracking under an unit discharge of $q=50 \mathrm{cms} / \mathrm{m}$. Figure 3(a), (b) and (c) are the particle trajectories for a group of particles with particle diameter $D$ equal to $400 \mathrm{~mm}, 50$ $\mathrm{mm}$ and $10 \mathrm{~mm}$, respectively. Comparing Figure 3(a)-(c), it appears the trajectory of the smaller particles tend to be more confined and move along the flow lines, while the large particle may bounce a few times after passing the knickpoint with a head drop.

Figure 4 shows the distribution of impact energy head for the case of $q=50 \mathrm{cms} / \mathrm{m}$ : Figure 4(a) for $D$ $=400 \mathrm{~mm}$ and Figure 4(b) for $D=10 \mathrm{~mm}$. The sequential spikes indicate the sequential occurrence of particle bounces. The location of each spike in the distribution corresponds to a concentrated region that particles may pass the knickpoint and hit the surface of groundsill. It appears the distribution of impact points for a larger particle tends to be more random than that of a small particle. A small particle passing the head drop tends to impact the ground 
surface at more concentrated regions and with less bounces; their impact points are more concentrated at a narrow band. The occurrence of concrete damage due to particle impact can be evaluated by the impact energy. Khali et al. (2015) conducted a series of laboratory experiments to determine the maximum impact energy that a standard grade concrete can take before its breakage; their test results indicated that an intact concrete will break when the accumulative impact energy is over $600 \mathrm{~J}$, approximately. For simplicity, $600 \mathrm{~J}$ can be regarded as the threshold impact energy.

The input of the impact energy may directly determine whether a single particle impact can result in damage to the attacked material. Assuming the same impact velocity, a large particle will certainly possess higher impact energy for each occurrence of impact. Setting $600 \mathrm{~J}$ as the threshold, one can determine the threshold impact velocity to damage a standard grade concrete for a particle with a specific particle size $D$. For a particle with $D=400 \mathrm{~mm}$, a vertical component of impact velocity larger than 3.8 $\mathrm{m} / \mathrm{s}$ is enough to damage a common concrete in one single impact. A particle passing the knickpoint can bounce a few times before its kinetic energy is dissipated.

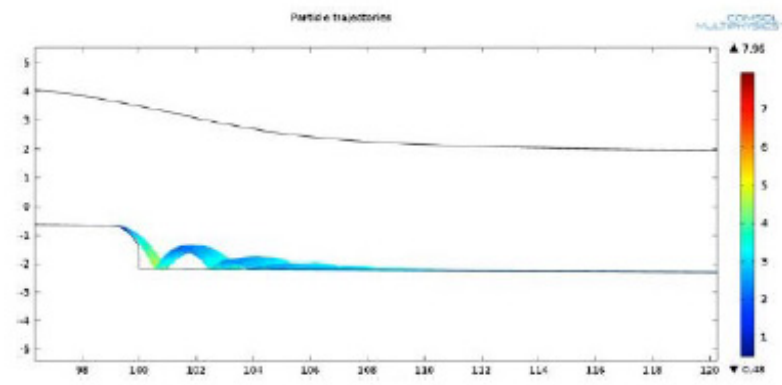

(a)

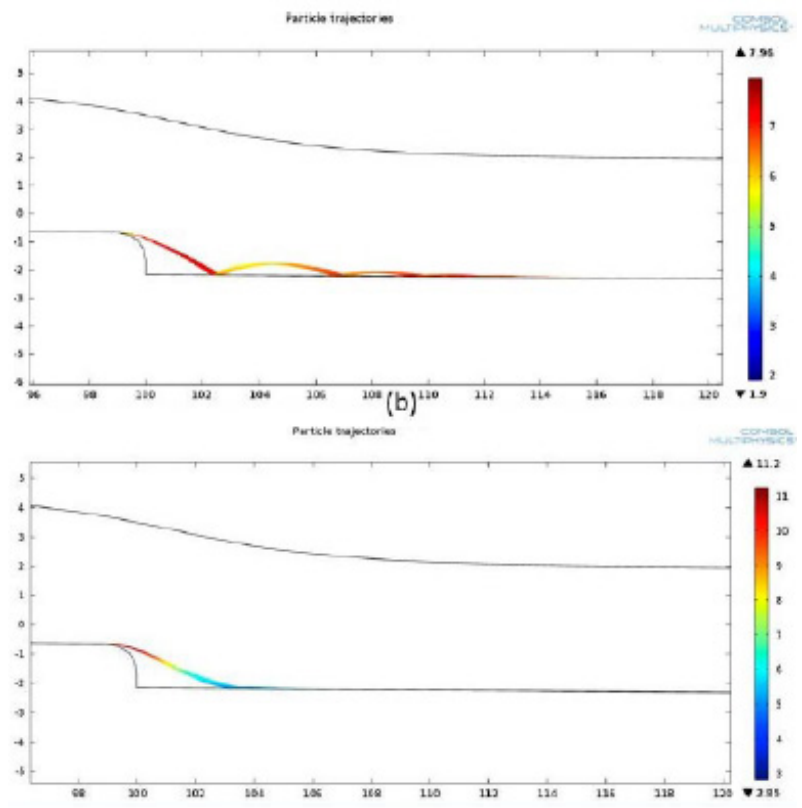

(c)

Figure 3. Particle trajectories for $\mathrm{q}=50 \mathrm{cms} / \mathrm{m}$ and $\mathrm{D}=(\mathrm{a})$ $400 \mathrm{~mm}$; (b) $50 \mathrm{~mm}$; and (b) $10 \mathrm{~mm}$.

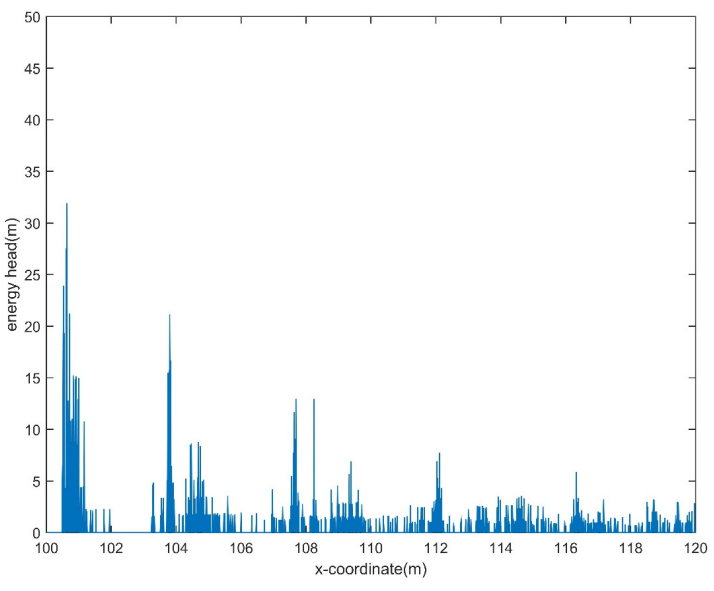

(b)

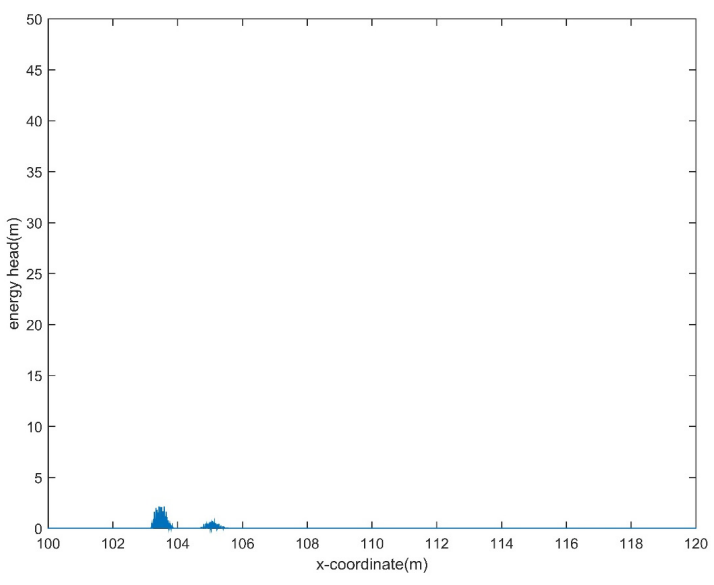

Figure 4. Impact energy-head distribution for the case $q=50$ $\mathrm{cms} / \mathrm{m}$ and for (a) $\mathrm{D}=400 \mathrm{~mm}$ and (b) $\mathrm{D}=10 \mathrm{~mm}$.

Table 1 lists the impact energy of successive bounces of a $400-\mathrm{mm}$ particle under various unit discharges. In the first collision, the impact energy exceeds the threshold impact energy and is able to cause concrete damage in just one impact. The impact energy gradually reduces as the particle continuously bounces away from the knickpoint. After the first collision, the impact energy is no longer large enough to break the concrete in a single impact. Nevertheless, repetitive impacts from sequential particle impacts during a flood may accumulate the impact energy at the same spot. As time elapses, the accumulated impact energy may eventually reach the threshold energy that causes breakage of the concrete. Smaller particles cause lower impact energy for an individual impact. However, the trajectory of small particles is more concentrated and more easily results in continuously impact at the same spot, and thus, may still result in damage accumulation and lead to concrete breakage.

Table 1. Impact energy of successive collisions of a 400-mm particle under various unit discharges.

\begin{tabular}{|c|c|c|c|c|}
\hline & $1^{\text {st }}$ & $2^{\text {nd }}$ & $3^{\text {rd }}$ & $4^{\text {th }}$ \\
\hline $50 \mathrm{cms} / \mathrm{m}$ & $731 \mathrm{~J}$ & $335 \mathrm{~J}$ & $152 \mathrm{~J}$ & $72 \mathrm{~J}$ \\
\hline $25 \mathrm{cms} / \mathrm{m}$ & $706 \mathrm{~J}$ & $351 \mathrm{~J}$ & $168 \mathrm{~J}$ & $78 \mathrm{~J}$ \\
\hline $10 \mathrm{cms} / \mathrm{m}$ & $665 \mathrm{~J}$ & $310 \mathrm{~J}$ & $147 \mathrm{~J}$ & $72 \mathrm{~J}$ \\
\hline
\end{tabular}


A particle moving in water current is simultaneously subjected to the gravitational force and the drag force resulting from the water flow. The kinematics of a particle is determined by these two forces. For a particle moving in an open channel, the drag force is affected significantly by the horizontal particle velocity; while the gravitational force controls the vertical particle velocity.

One can convert the acting forces into equivalent acceleration terms. The gravitational acceleration $g$ is assumed a constant. The drag force resulting from water flow is proportion to the acting area, thus it is proportional to $\mathrm{D}^{2}$, while the particle mass is proportional to $\mathrm{D}^{3}$. Hence, the equivalent acceleration for a larger particle from the drag force will be lower than that for a small particle.

Figure 5 summarizes the equivalent accelerations. The horizontal equivalent acceleration for the drag force is proportional to $\mathrm{C}_{\mathrm{x}} / \mathrm{D}$, where $\mathrm{C}_{\mathrm{x}}$ is the coefficient of horizontal drag force. The vertical equivalent acceleration can be expressed as $\mathrm{g}+\mathrm{C}_{\mathrm{y}} / \mathrm{D}$, where $\mathrm{C}_{\mathrm{y}}$ is the coefficient of horizontal drag force.

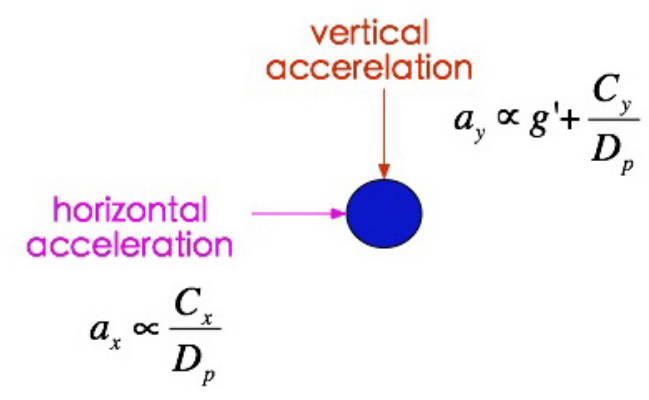

Figure 5. The horizontal and vertical equivalent accelerations of a particle moving with water flow

For a relative large particle, the gravitational force tends to dominate the behaviour of the particle movement; the importance of the drag force is relative smaller, and thus a second order effect. In contrast, the drag force has a stronger effect on the kinematics of a relatively small particle compared to the effect due to the gravitational force. A small particle more or less travels with the water flow, so that the particle trajectory closely follows the flow lines.

The intensity of the discharge affects the characteristics of particle movement. A higher discharge can carry a higher kinetic energy in the water flow, thus enabling the transportation of larger particles past the weir. Once a large particle passes an elevation drop, the loss of potential energy will be transferred to kinetic energy. For example, a 400-mm particle leaving the head drop possesses a high downward vertical force. As a result, there will be a high vertical component of downward acceleration as well as a high vertical component of impact velocity. Consequently, the impact angle for a larger particle is usually larger than the impact angle of a small particle.
For a lower flow discharge, only relatively small particles can move with the water flow; the drag force on the particle is also lower. Thus, the horizontal component of particle velocity is lower and results in an even higher impact angle as a result. Although the horizontal component of impact velocity is controlled by the flow discharge, the vertical component of impact velocity is insignificantly affected by the magnitude of discharge.

\section{CASE STUDY}

In this section, we present a case study of groundsill damage due to the impacts of moving pebbles in the Ba-Chang River, Taiwan. The Ba-Chang River is one of the major rivers in southwestern Taiwan. The site of the case study is located at the midstream of this river. Particles as large as $400 \mathrm{~mm}$ were observed being transported downstream during large floods. Figure 6 shows two photographs at the case study site. Figure 7 illustrates the layout of the groundsill works.
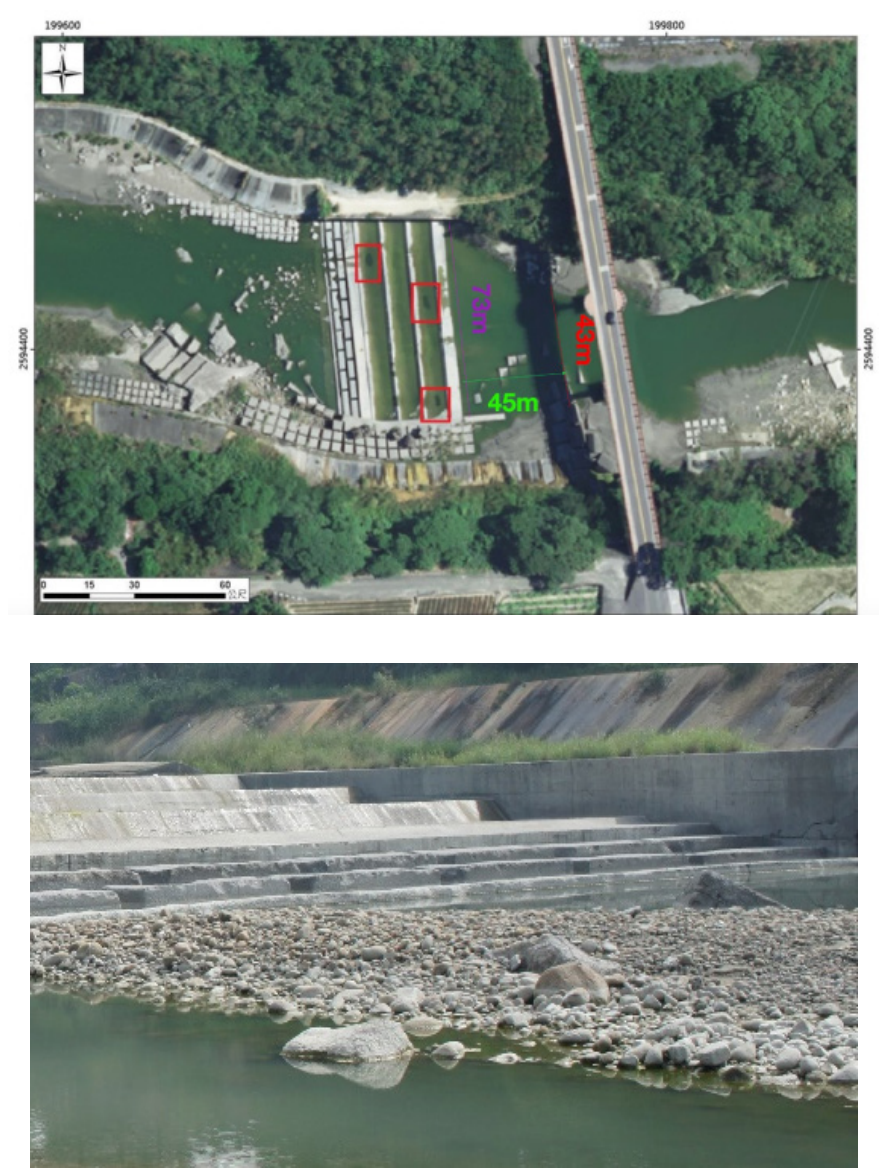

Figure 6. Photographs at the site of the case study

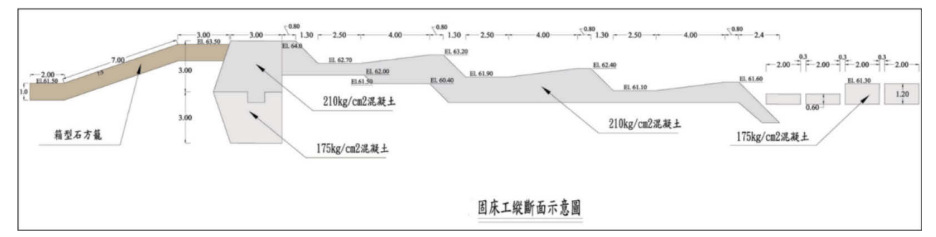

Figure 7. Layout of the groundsill 
Figure 8(a) displays the two-dimensional FEM model mesh along the river section with a series of stepped groundsills. The system of the stepped groundsills contains one weir on the leftmost, followed by three successively descending steps. The unit discharge, $q$, is $476 \mathrm{cms} / \mathrm{m}$, this discharge corresponds to a discharge with a two-year return period. The slope in this river section is $1 / 150$, approximately.

Figure 8(b) shows the flow field obtained from the CFD calculation after convergence. We also examined the tractions arising from water flow by means of a fluid-structure interaction analysis. According to the results of the fluid-structure interaction analysis, all of the components of the tractions are less than a few tens of $\mathrm{kPa}$, which are far lower than the concrete strength (usually a few to tens of $\mathrm{MPa}$ ) for any mode of failure (the results are not shown). It reveals that water flow alone is not able to damage the concrete making up the groundsill.

(a)

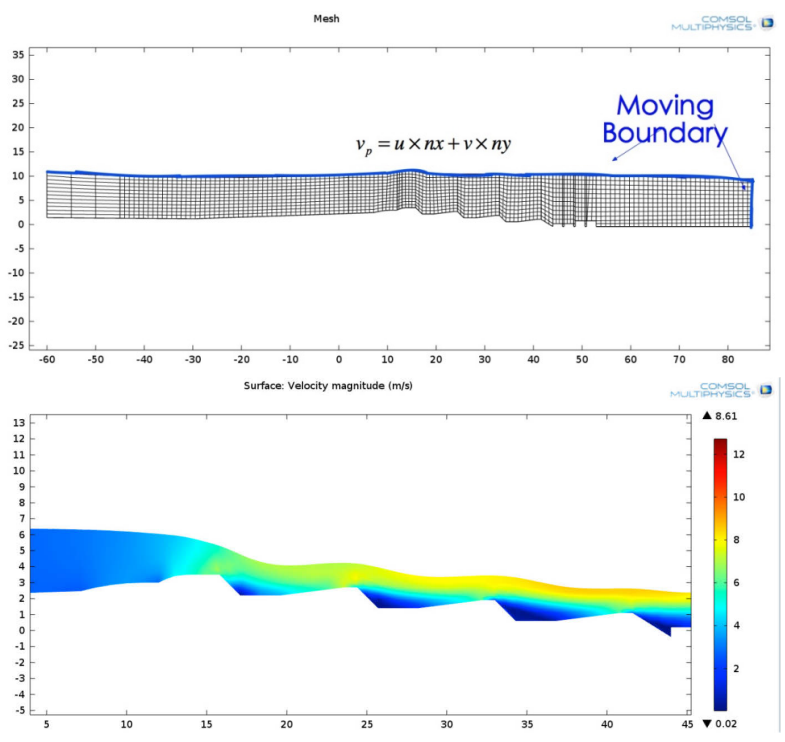

Figure 8. FEM model and the flow field of the case study

Figure 9 displays the distribution of particle trajectories and velocity for this case study. The regions of the impact concentration occur at approximately halfway along the three steps downstream of the weir. The impact velocity decreases with the descending steps.

The particle tracking simulation follows the analysis of flow field. For the two-year return period discharge, particles up to $100 \mathrm{~mm}$ in diameter are able to pass through the series of stepped groundsill. Figure 10 shows the impact energy-head distribution obtained from the particle tracking for particles with $100 \mathrm{~mm}$ diameter. It appears that the spots with high impact-energy head are mainly located adjacent to each elevation drop. The highest impact-energy head obtained from the simulation coincides with the locations of damage observed in the field as shown in Figure 11.

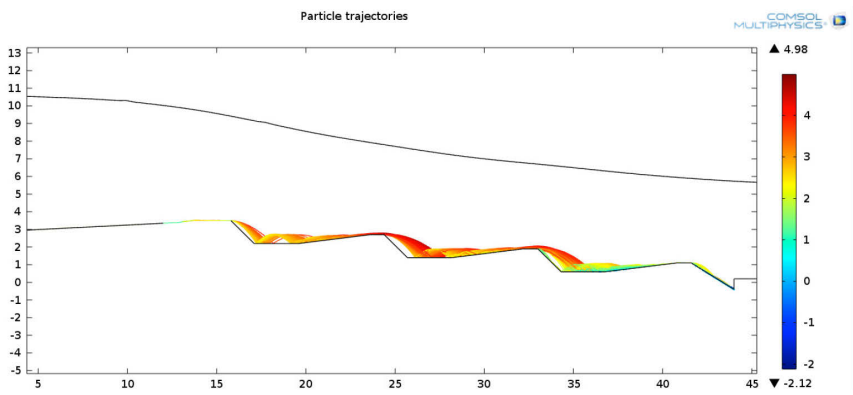

Figure 9. Distribution of particle trajectories for the case study

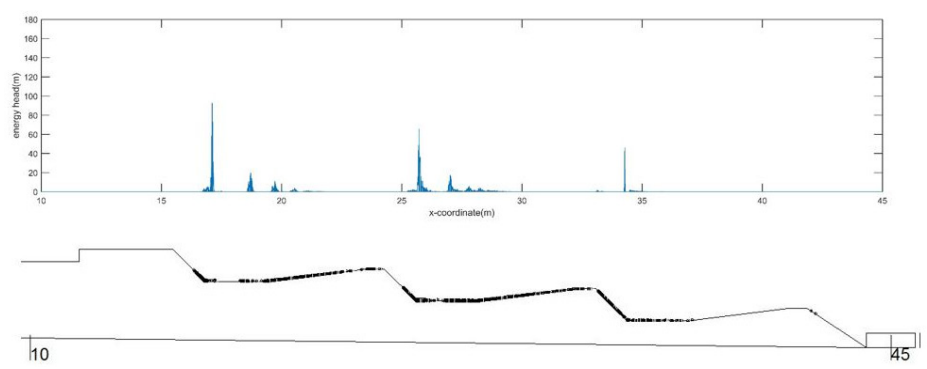

Figure 10. Impact energy-head distribution for the case study
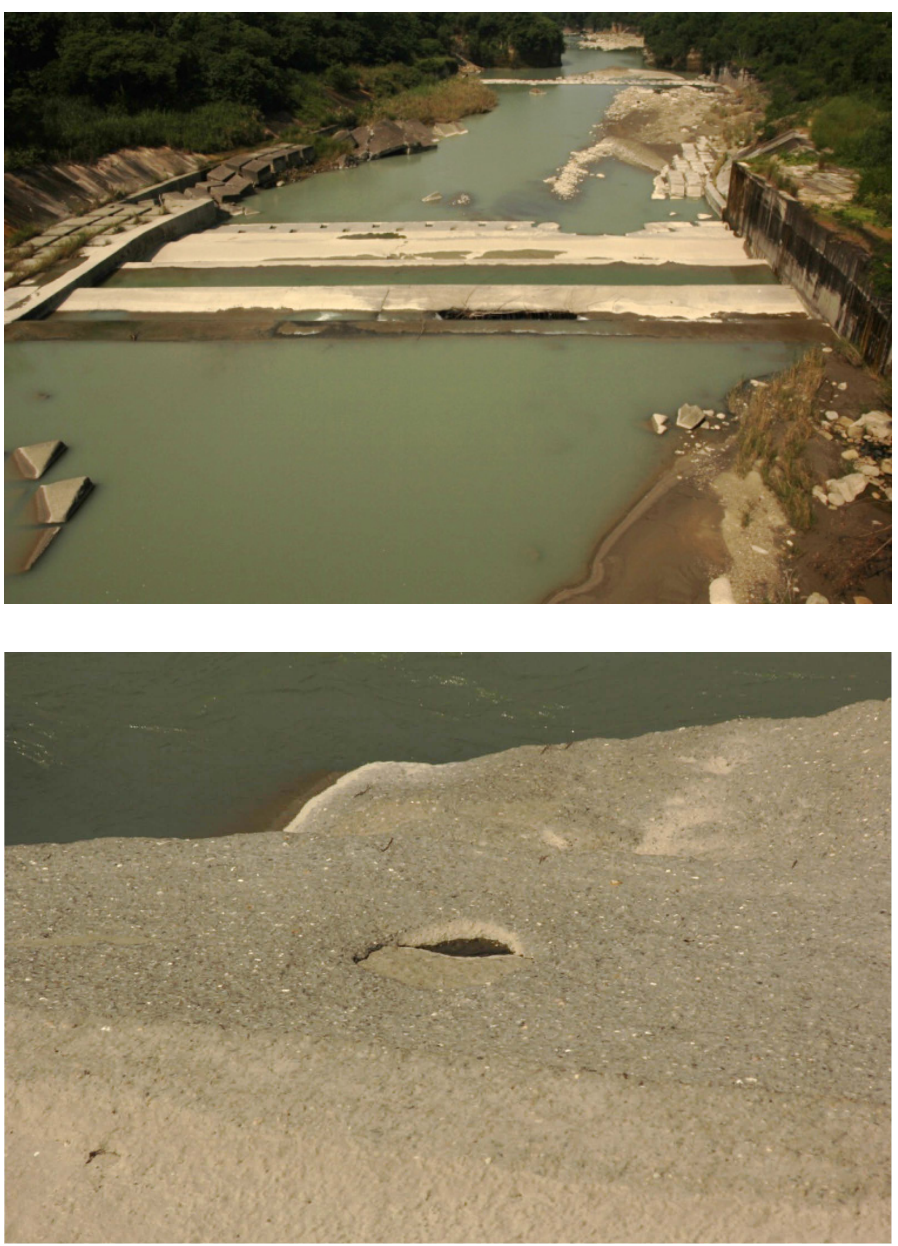

Figure 11. Photographs showing the damage of groundsill

The impact load and stresses due to each impact can be assessed by the Hertz theory. The impact load $F$ was estimated from the normal component of the impact velocity $v_{\mathrm{o}}$ by using $F=k \cdot v_{\mathrm{o}}{ }^{1.2} \cdot R^{2}$; the term $k$ is a proportionality coefficient. The likelihood of concrete failure caused by particle impact can be assessed by examining the maximum impact energy or 
the maximum impact stress on the concrete. Using the Boussinesq solution (Das \& Sobhan, 2014), one can estimate the induced stress caused by a concentrated load on a semi-infinite space at a distance from the acting load. The maximum depth $z$ at the location where the induced stress will exceed the concrete strength then can be determined. This depth, $z$, can be regarded as the maximum depth of concrete breakage caused by one impact load.

Table 2 lists various impact variables of 100$\mathrm{mm}$ particles on steps 1, 2 and 3 of the groundsills. In the Table, $v_{p}$ is the particle velocity; $v_{n}$ is the vertical component of $v_{p} ; F / W$ is the impact load $F$ normalized by the particle weight $W$; and $z / D$ is the depth of concrete breakage $z$ normalized by the particle diameter $D$. The principles in the ACI544 test (1999) and the dynamic strength of concrete (Chen, 2015) are used to examine whether the groundsill will fail and to estimate the breakage depth in the concrete.

Table 2. Data of Impact Variables

\begin{tabular}{|c|c|c|c|c|c|}
\hline $\begin{array}{c}\text { Groundsill } \\
\text { Step \# }\end{array}$ & $\begin{array}{c}V p \\
(\mathrm{~m} / \mathrm{s})\end{array}$ & $\begin{array}{c}V n \\
(\mathrm{~m} / \mathrm{s})\end{array}$ & $\begin{array}{c}\text { Impact } \\
\text { angle }\end{array}$ & $F / W$ & $z / D$ \\
\hline 1 & 3.60 & 2.02 & $34.04^{\circ}$ & 175.09 & 0.074 \\
\hline 2 & 2.94 & 1.83 & $38.40^{\circ}$ & 159.46 & 0.059 \\
\hline 3 & 1.43 & 0.94 & $41.23^{\circ}$ & 70.91 & 0.04 \\
\hline
\end{tabular}

\section{CONCLUSIONS}

A pebble or cobble passing a head drop can carry a large impact momentum when it drops and hits the groundsill. The heavy impact may result in the breakage of the concrete. The broken areas in concrete may gradually expand and cause the failure of the groundsill, eventually. This study has explored this problem using CFD and the FEM software COMSOL. The numerical simulation consists of two steps. The first step involves an open channel simulation of turbulent flow with a free water surface; its purpose is to obtain the flow filed under a specific input discharge on the inlet boundary. With the calculated flow field, the next step is to model the paths of particles (i.e., particle tracking) to be carried by the flow field; the forces acting on a particle include the gravity force and the drag force in the flow.

The simulated results reveal that the kinematics of a single particle passing a head drop is primarily affected by the size of particles. A small particle tends to travel along the flow lines and brings relatively low impact energy; the impact location is more confined. A large particle, on the other hand, is more affected by gravity; saltation is more likely and will carry relatively high impact energy. The magnitude of the hydraulic event controls the discharge magnitude of river. The major role of the discharge is to enable the transportation of larger particles to the midstream, and even downstream of a river.
The impact position, the impact velocity and the impact angle for each impact are recorded during the numerical simulation of particle tracking. With these recorded data, it is then possible to evaluate and carry out the statistics of the impact position, impact load and impact kinetics. The locations prone to damage can be evaluated. The Hertz theory is adopted to estimate the impact load and stresses on the concrete surface due to an impact. The maximum impact energy or impact stress determines whether a particle impact will result in the failure of concrete.

The findings from this study help to understand the kinematics and mechanics of gravel particles travelling in a river in strong water flow. It may also be used to predict the potential extent and locations of groundsill damage under a major hydraulic event.

\section{REFERENCES}

Chen, X., Wu, S. and Zhou, J. (2015). "Large-Beam Tests on Mechanical Behavior of Dam Concrete under Dynamic Loading", J. Mater. Civ. Eng.

COMSOL (2013) CFD module user's guide.

COMSOL (2014) Particle Tracking Module user's guide.

Das, B.M. and Sobhan, K. (2014) Principles of Geotechnical Engineering, 8th Ed., Cengage Learning

Donea, J., Giuliani, S. and Halleux, J.P. (1982) "An arbitrary Lagrangian-Eulerian finite element method for transient dynamic fluid-structure interactions", Computer methods in applied mechanics and engineering, 33(1), pp. 689-723.

Hertz, H.R. (1882) "Ueber die Beruehrung elastischer Koerper (On Contact Between Elastic Bodies)", in Gesammelte Werke (Collected Works), Vol. 1, Leipzig, Germany, 1895.

Huang, M.W., Pan, Y.W. and Liao, J.J. (2012) "A case of rapid rock riverbed incision in a coseismic uplift reach and its implications", Geomorphology, 184, pp. 98-110.

Huang, M.W., Liao, J.J., Pan, Y.W., Cheng, M.H., (2013) "Failure Mechanisms of the Vertical Drop Structures in Weak Bedrock Channel", The 23rd International Ocean and Polar Engineering Conference (ISOPE-2013), Anchorage, Alaska, USA, pp. 716-721

Huang, M.W., Liao, J.J. and Pan, Y.W. (2014) "Rapid channelization and incision into soft bedrock induced by human activity - implications from the Bachang River in Taiwan", Engineering Geology, 177, pp.10-24.

Khalil, E., Abd-Elmohsen, M. and Anwar, A.M. (2015) "Impact Resistance of Rubberized Self-Compacting Concrete", Water Science, 29, pp. 45-53.

Kleis, I. and Priit K. (2008) "Solid particle erosion." Occurrence, Prognosification and Control.

Kraai P, P. (1998) "ACI-544, measurement of properties of fiber reinforced concrete" Materials Journal, 85(1), pp. 45-65.

Plum, D. and Fang Xu. (1996) "A rock and a hard place." International water power \& dam construction, 48(7), pp. 3033. 